\title{
Abandono escolar, Éxito académico y Género: abriendo caminos desde la interseccionalidad para la inclusión y la justicia
}

\author{
Rosa Vázquez Recio ${ }^{a}$, Guadalupe Calvo García ${ }^{b}$, Mónica López Gil ${ }^{\mathrm{c}}$ \\ ${ }^{\mathrm{a} U n i v e r s i d a d ~ d e ~ C a ́ d i z ~(r m a r i a . v a z q u e z @ u c a . e s), ~}{ }^{\mathrm{b}}$ Universidad de Cádiz \\ (guadalupe.calvo@uca.es), ${ }^{\mathrm{C} U n i v e r s i d a d ~ d e ~ C a ́ d i z ~(m o n i c a . m a r i a @ u c a . e s) ~}$
}

\begin{abstract}
Resumen
El presente trabajo aborda uno de los temas que resulta de mayor interés en los últimos tiempos, a nivel nacional como internacional, el denominado abandono educativo. Este constructo resulta necesario analizarlo en su relación con él éxito académico, pues ambos responden a formas de entender la excelencia en el marco de las jerarquías (sociales, educativas, culturales, etc.). En este caso en concreto nos interesa realizar su análisis desde una mirada interseccional, en la que sin duda el género juega un papel clave, sin olvidar su interrelación con otra serie de categorías que ayudan a definir y a comprender las situaciones de privilegio como de discriminación, exclusión e inequidad. El análisis lo realizamos desde los aportes de la investigación cualitativa realizada, centrándonos en las voces de las adolescentes y jóvenes que han abandonado el sistema educativo. Concluimos con una serie de reflexiones para la práctica.
\end{abstract}

Palabras clave: abandono escolar, éxito educativo, género, adolescentes

\section{Introducción}

En los últimos tiempos, uno de los temás que resulta de interés y suscita gran preocupación, tanto a nivel nacional como internacional, es el abandono educativo. Las tasas, con carácter general y sin entrar en las particularidades de cada país, han disminuido. No obstante, y pese a ello, sigue siendo una problemática a la que atender. El abandono escolar es un constructo que necesita ser analizarlo en su relación con él éxito académico, pues ambos responden a formas de entender la excelencia en el marco de las jerarquías diversas (sociales, educativas, culturales, etc.). En este caso en concreto nos interesa realizar su análisis desde una mirada interseccional, en la que sin duda el género juega un papel clave, sin olvidar su interrelación con otra serie de categorías que ayudan a definir y comprender las situaciones de privilegio así como de discriminación, exclusión e inequidad. La interseccionalidad (Hill Collin, 1990, 2009, Hill Collins y Bilge, 2019) se nos presenta 
como una herramienta de análisis crítico con la que poder contribuir a la inclusión, equidad y justicia. El análisis se realiza desde los aportes de la investigación cualitativa realizada bajo el proyecto "Riesgo de fracaso y abandono escolar en Secundaria Obligatoria. Los contextos educativos, familiares y socioculturales. Un estudio cualitativo" (P12-SEJ2664, Junta de Andalucía), cuyo propósito central era analizar los factores que resultan determinantes para que la población adolescente llegue a estar en situación de riesgo de fracaso y abandono escolar, desde las voces de los propios protagonistas. Este trabajo se ha focalizado en las voces de las chicas que han abandonado el sistema educativo. Sus experiencias nos ayudan a visibilizar la importante incidencia de las normas de género sexistas en su desarrollo académico, profesional y personal.

\subsection{Referentes sin voces, sin cuerpos}

El fracaso escolar y el abandono educativo son dos asuntos que suscitan una gran preocupación y se reconocen como un problema público y social (Rujas Martínez-Novillo, 2017), nacional e internacional, a los que las políticas educativas intentan dar solución. El abandono escolar temprano se sitúa en España en un 17,3\% y en Andalucía, en un 21,6\%. Desagregados los datos por sexos y atendiendo al tramo de edades comprendidas entre los 18 a 24 años, el 13\% de las mujeres y el 21,4\% de los hombres han abandonado el sistema educativo prematuramente en 2019 (Ministerio de Educación y Formación Profesional, 2020). La literatura sobre el tema es muy amplia e identifica, entre los factores que intervienen, el género. Los trabajos que abordan estos fenómenos desde la perspectiva de género suelen hacer hincapié en la mayor incidencia de los mismos en la trayectoria de los chicos, o en las mayores tasas de éxito escolar de las chicas (Eurostat, 2018; European Commission. 2019). Según lo expuesto, cabe pensar que el éxito es consustancial a las chicas, y que ellas, dentro del sistema educativo, siempre lo van a alcanzar. Si se admite tal supuesto, dicho éxito escolar enmascara los obstáculos y las dificultades derivadas de estructuras organizativas patriarcales y de un currículum que sigue privilegiando ciertas voces, entre las que no estarían preferentemente las de ellas y, aún menos, las de aquellas que no pertenecen al grupo hegemónico (musulmanas, negras, gitanas, lesbiansa, trans, etc.). Las estudiantes fracasan y abandonan como consecuencia de esas dificultades y esos obstáculos que el sistema educativo, aparentemente inclusivo, les acarrea. Son triunfadoras por sus tasas de éxito en los diferentes niveles educativos (Martínez García, 2007, 2011; García, Padilla, y Suárez, 2009; Rodríguez-Martínez y Blanco García, 2015; Eurostat, 2018; European Commission, 2019), pero no dejan de experimentar y sufrir las desigualdades que un sistema educativo clasista, patriarcal y colonialista promueve. Al mismo tiempo, debido al modelo de feminidad asentado en el imaginario colectivo, las buenas calificaciones llegan a ser atribuidas más a la suerte o a factores que no tienen que ver directamente con su capacidad y potencial, a diferencia de lo que ocurre cuando no son 
buenas (Rodríguez Martínez, 2011, p. 80). El éxito también se explica como efecto de las características identificadas y atribuidas a ellas (pasivas, dóciles, dependientes, actitud de ayuda a los demás, responsables, obedientes, tranquilas, etc.) que resultan favorables para adaptarse a las exigencias de una escuela en la que el orden, el control, la vigilancia y sus dispositivos de acción, propios de la tecnología disciplinaria, son clave (Foucault, 1981). Así visto, las chicas resultan ser las deseadas para la sociedad del rendimiento que tenemos, a la que solo interesan los sujetos mejores, los emprendedores exitosos y los más competitivos (Han, 2012): necesitan chicas de rendimiento, y pese a que las investigaciones señalan la importancia de sentirse parte de un grupo (Skelton, 2010), ellas son las más exitosas en una escuela que fomenta la competitividad. Por esta regla, parece que no es conveniente que realmente abandonen de modo definitivo el modelo de feminidad impuesto social e históricamente, algo que parece mantenerse (Rodríguez y Megías, 2015) pese a las conquistas que se han dado en igualdad y equidad entre géneros. Sin embargo, no podemos olvidar, como recoge Julià (2018), que muchas chicas, entre otros motivos que podemos considerar, desean seguir estudiando para alcanzar una vida independiente y autónoma (Rodríguez Martínez y Blanco García, 2015) y para evitar caer en la trampa del trabajo doméstico. De alguna manera, los chicos se sienten menos presionados al respecto (Martínez García, 2007) por los roles tradicionales asignados a ellos. Valgan, como ejemplo, los datos ofrecidos por el INE (2020), con respecto al criterio "personas inactivas según razones de la inactividad y grupos de edad", en el tramo de edad de 15 a 24 años (el abandono educativo prematuro se fija entre los 18 y 24 años): el 2,1\% de las mujeres frente al 1,6\% de los hombres lo están por responsabilidades familiares o personales, y el 1,4\% de las mujeres, frente a $0 \%$ de hombres, se encuentran en dicha situación por cuidado de niños/as, adultos enfermos, discapacitado o mayores.

\subsection{La metodología asumida en nuestra investigación}

Siguiendo los propósitos de nuestro proyecto, antes indicado, en esta comunicación queríamos hacemos hincapié en la perspectiva de género. Nuestro objetivo es ver en qué medida las normas de género dominantes condicionan la trayectoria académica de las chicas, empujándolas al fracaso y/o al abandono escolar, pese a que se dé en menor proporción que en el caso de los chicos. En coherencia con las pretensiones, se asumió una metodología cualitativa. Las estrategias de recogida de información fueron: entrevistas semiestructuradas (91), estudios de casos biográficos (9) y grupos focales (3), y se han llevado a cabo en Cádiz, Málaga y Almería. Las edades han estado comprendidas entre los 12 y los 26 años. Se aplicó para el tratamiento de los datos Nvivo11. Para este trabajo nos hemos centrado en los relatos de las 18 jóvenes entrevistadas en Cádiz. El motivo de centrarnos en esta provincia responde a que ha sido donde hemos obtenido la mayor muestra. La triangulación se ha realizado a partir del contraste de las experiencias de cada 
Abandono escolar, Éxito académico y Género:

abriendo caminos desde la interseccionalidad para la inclusión y la justicia

una de las chicas y de las mismas con diferentes aportaciones teóricas acerca de desigualdades vinculadas al género que tienen lugar dentro y fuera del ámbito escolar. Finalmente, el anonimato se ha garantizado con nombres ficticios.

\subsection{Hablan ellas, hablan sus experiencias}

El análisis de los datos nos permite establecer dos grupos atendiendo a la motivación hacia el estudio y el aprendizaje que tienen las jóvenes participantes. Por una parte, se encuentra el grupo de las chicas que presentan una motivación extrínseca con una visión instrumental de la educación, perspectiva que, según Cerezo y Casanova (2004), es más propia de los chicos, más tendentes al fracaso, como fue mencionado antes. Por otra, las jóvenes con motivación intrínseca por el aprendizaje, asociada por los mismos autores, sobre todo, al alumnado femenino; que fracasaron inicialmente en la escuela y/o llegaron a abandonarla por diversas situaciones complicadas, pero que disfrutan aprendiendo y quieren crecer como personas. En el primer grupo podríamos incluir las jóvenes que, independientemente de si en la actualidad están estudiando o no, consideran que el título es la llave para poder acceder a un empleo, y en el segundo, aquellas a las que les gusta aprender.

\section{a) ¡Educación, dame empleo, aunque entre medias tenga que resistir!}

Ana estudió un PCPI (Programa de Cualificación Profesional Inicial) de estética con intensión cursar un Ciclo Formativo de Enfermería animada por su madre que trabaja como limpiadora en un hospital, porque sabe que los contactos de esta le facilitarán el acceso a un puesto de trabajo. Jimena, que rechaza todo lo que tiene que ver con el instituto, realiza unas prácticas en una escuela infantil con intención de seguir estudiando una titulación superior para poder desarrollar este trabajo que le interesa; su otra opción, que no descarta, es peluquería. A Raquel le gusta el mundo de la estética y la peluquería, y considera que con ambas podrá tener un acceso al empleo sin muchas dificultades, pues puede ejercerlas como particular, sin depender de ningún contratante. En esta misma línea formativa y de proyección futura se encuentran Belén y Dina. Por su parte, Caty no terminó el PCPI de comercio tras quedarse embarazada; estudia ESPA (Educación Secundaria para Personas Adultas) y su objetivo es acceder al cuerpo de policía, si bien no descarta otras opciones, como estudiar hostelería. Por último, Geli, quien también abandonó los estudios de ESO a causa de su embarazo, pese a que ha realizado varios intentos de estudiar ESPA, lo único que busca es tener dinero para poder independizarse con su pareja y su hija; espera que le ofrezcan participar en una escuela-taller o en algún otro curso que le ayude a entrar en el mucho laboral.

Estas chicas, con proyección de futuro, identifican el comienzo de su rebeldía y/o desmotivación hacia los estudios en el paso al instituto, tránsito que repertucte de modo 
diferente según caso (Camacho Ruiz, 2016). Ana explica que, cuando accedió al instituto, no le gustó nada; expone que en esa época se volvió una "contestona" y que se aburría en clase, llegando a dormirse (a pesar de que todo esto le avergonzaba al mismo tiempo). Ester achaca su abandono de la escuela a su juventud y se autocalifica de "torpe", motivo por el que dice que le cuesta mucho avanzar en los estudios; ello y el comienzo de la relación con su novio, que tampoco estudiaba, la llevó a faltar; cursa ESPA animada por toda su familia. Para Jimena el instituto es una incógnita a la que no le encuentra sentido; desde sus inicios comenzó a recibir abundantes partes de incidencia, reconoce que a veces se ha dormido en clase y que cualquier actividad le resulta más motivadora que el estudio (el portátil, el móvil, charlar con su abuela, bailar hip hop, etc.). Por su parte, Raquel se rebeló en $2^{\circ}$ de ESO al ver que cada curso aumentaba la dificultad y experimentar un bloqueo ante los exámenes; al igual que Ester, se prepara la ESPA, aunque se siente torpe e incapaz de tener éxito sin el apoyo del profesorado que se preocupa por ella.

Tanto Belén como Caty experimentaron la rebeldía y la desmotivación en su adolescencia, pero el punto de inflexión en su escolaridad fue cuando fueron expulsadas temporalmente del instituto. Caty, además, añade como motivo la relación que mantuvo con un chico hasta sus 15 años, que terminó con toda su vida social. Para Belén, su situación se hace más complicada cuando empieza a frecuentar un grupo de chicas y chicos que nunca iban a clase y consumían porros; en esa etapa conoció al que sería el padre de su hija. En esa época, ella vivía con su padre, pero lo dejó para irse con la familia de su pareja una vez que se quedó embarazada; la vida en casa de su pareja le resultó un infierno, ya que este se emborrachaba y drogaba, e incluso llegó a agredirla. Tras cuatro años allí, llegó a pesar 39 kilos y debió ser internada en un centro para desengancharse de los porros. Consiguió abandonar este contexto para volver a vivir con su padre, con su hija y con su nueva pareja, considerado el "salvador de su vida". Geli, que se valora como torpe, considera que fue su embarazo el punto crítico que la llevó a abandonar el instituto; si bien, hay otros motivos a añadir: ser sus progenitores drogadictos, tener que vivir con su abuela junto a sus hermanos (en total son nueve) y experimentar el rechazo de sus iguales de la escuela por estas circunstancias (realmente, eran los padres quienes prohibían a sus hijos e hijas jugar con ella). Para Dina lo determinante fue su repetición de curso; se desanimó al verse rodeada de "niños pequeños" y dejó de estudiar. De estas experiencias, merece prestar atención a dos aspectos centrales: sus relaciones sentimentales y la falta de confianza en sus capacidades intelectuales.

Respecto a las relaciones amorosas, hemos anticipado algo sobre los efectos de estas en los estudios de Ester, Belén y Caty. Podemos afirmar que las dos últimas han sido víctimas de violencia machista, ya que Belén llegó a sufrir agresiones físicas y Caty llegó a encontrarse totalmente aislada. Pero también Ana llegó a estar ingresada en el hospital con anemia debido a que no comía porque su novio la llamaba "gorda"; desde esta experiencia no 
quiere tener pareja. Por su parte, Ester, en parte, dejó de estudiar porque su novio tampoco lo hacía. Geli señala que le gustaría que alguien asistiera con ella a la escuela y la animara, y aunque se lo ha propuesto en diversas ocasiones a su pareja, no lo consigue debido a que no tiene ningún interés. En otra línea, Raquel continúa estudiando centrada en conseguir un trabajo que le permita independizarse con su novio. Respecto a la percepción de sus propias capacidades intelectuales, está extendida la concepción "torpe" e "incapaz" entre la mayoría de las chicas. Por último, cabe señalar que la mayoría de estas jóvenes reconocen realizar labores de cuidados y domésticas (típicamente femeninas), tanto en sus propios hogares como en los de otras personas, a modo de salida laboral (cuidado de hermanos pequeños, de primo, de abuela, de mujer anciana, limpiando escaleras, etc.).

\section{b) ¡Educación, dame: tengo ganas de aprender!}

En el segundo grupo que identificamos bajo la idea de la motivación intrínseca, con ganas de aprender, se detectan diferencias, inicialmente, en sus hobbies y actividades de ocio; estas se orientan al deporte, la música, la cultura y también a la acción social (bailar, dibujar, tocar el piano y el trombón, la lectura, el teatro, la caracterización, voluntariado en una asociación, etc.). Desde estas referencias cabe preguntarse por qué tuvieron dificultades en la escuela, llegando a abandonarla. En esta ocasión las razones son bien diversas: la mayoría ha sido marcada por experiencias de bullying y exclusión (insultos como "gorda", "bigote", "piojosa", "marimacho", por tener padre gitano, falta de recursos económicos, agresiones), la falta de apoyo en diversos sentidos, las separaciones de sus progenitores, los fallecimientos de seres queridos, los enfrentamientos con el profesorado, la asunción de responsabilidades domésticas y de cuidado propias de la etapa adulta, o las ganas de disfrutar de la vida que, al final, desembocó en situaciones contrarias (consumo de drogas, depresión, pérdida de amistades, etc.). Afortunadamente, nuestras chicas se han crecido ante las adversidades han tomado las riendas de sus vidas, y en la actualidad tienen proyectos para crecer como personas (como retomar los estudios y hacer peluquería, hacer un ciclo formativo en Educación Infantil, un Grado Medio de enfermería e ir a la Universidad para estudiar medicina, montar un negocio de confitería, llegar a ser la primera árbitra de su pueblo, trabajar en una pizzería para ahorrar y estudiar en Estados Unidos maquillaje de caracterización), y ello gracias al apoyo, en algunos casos de sus familias, y en otros de sus parejas.

\section{Conclusiones}

Las experiencias de estas jóvenes nos ayudan a visibilizar la importante y significativa incidencia de las normas de género sexistas en sus vidas, y de su influencia clave en el desarrollo académico, personal y profesional de ellas; pero también la influencia de una institución educativa que sigue marcada en sus elementos estructurales por el patriarcado. 
Estamos convencidas de la necesidad de continuar difundiendo modelos femeninos alternativos, diversos y no estereotipados. La tarea de dar visibilidad a las mujeres con físicos diferentes a los socialmente valorados, de diferentes etnias, culturas, religiones, orientación sexual, etc., debe ser prioritaria. Es fundamental enseñar que las mujeres pueden realizarse y tener una vida plena teniendo o no hijos e hijas, teniendo pareja (una, varias, del género que sea...) o sin tenerla. $\mathrm{Y}$ en esta misma línea, es preciso que para conseguir una igualdad afectiva se trabaje por una educación sentimental y sexual no romántica, no heteronormativa y no coitocéntrica, desde los primeros años de entra en las instituciones educativas. Una educación en la que se desidealice el amor de pareja y que se fomente relaciones sanas, igualitarias y libres. Igualmente, es fundamental acercar a los chicos al mundo de los cuidados. Mostrarles, igualmente, modelos de masculinidad no estereotipados. Liberarlos de la represión emocional a la que han sido sometidos y enseñarles a desenvolverse sin tener que recurrir a la violencia. Permitirles experimentar la gratificación que produce el cuidarse y el cuidar a otras personas $\mathrm{y}$, en definitiva, los beneficios de relacionarse en igualdad.

Los resultados de esta investigación tienen una clara y directa repercusión a dos nvieles: el primero, la formación inicial del profesorado se convierte en un ámbito prioritario para abordar las cuestiones planteadas; la perspectiva de género debe ser incorporada en la práctica docente del profesorado desde esa formación inicial para que de este modo se dispongan de las estrategias necesarias que permitan analizar, sin prejucios, estereotipos y sesgos de género en las realidades educativas. El segundo, la práctica investigadora, que no puede olvidar que "lo personal es político", y que todo intento de comprender las realidades para constribuir al cambio y a la transformación por un mundo sin discriminación, desigualdades e injusticias, requiere de un compromiso ético y político.

\section{Referencias}

Camacho Ruiz, Ma Auxiliadora (2016): Fracaso escolar y abandono educativo temprano en Educación Secundaria Obligatoria: un estudio integrado, Universidad de Huelva.

Cerezo, M $\mathrm{M}^{\mathrm{a}}$ T. y Casanova, P. F. (2004). Diferencias de género en la motivación académica de los alumnos de Educación Secundaria Obligatoria. Revista Electrónica de Investigación Psicoeducativa, 2 (1), 97-112. Recuperado de https://bit.ly/3gRUbBo

European Commission (2019, abril). Early leavers from education and training, Eurostat, Statistics Explained. European Commission. Recuperado de https://bit.ly/3dBm7aK

Eurostat (2018). Les femmes ont gagné en moyenne $16 \%$ de moins que les hommes dans l'UE en 2016. Recuperado de https://bit.ly/3cuTx9y

Foucault, M. (1981). Vigilar y castigar: nacimiento de la prisión (5 $5^{\text {a }}$ edic.). Madrid: Siglo XXI. 
Abandono escolar, Éxito académico y Género:

abriendo caminos desde la interseccionalidad para la inclusión y la justicia

García, S., Padilla, Ma T., Suarez, M. (2009). Los intereses académicos y profesionales de chicas que finalizan la escolaridad obligatoria. Revista de Educación, 349, 311-334.

Han, B. (2012). La sociedad del cansancio. Barcelona: Herder.

Hill Collins, P. (1990). Black Feminist Thought. Knowledge, consciousness and the politics of empowerment. London: Routledge.

Hill Collins, P. (2009). Another kind of Public Education. Race, Schools, the Media and Democratic Possibilities. Boston: Beacon.

Hill Collins, P. y Bilge, S. (2019). Interseccionalidad. Madrid: Morata.

Julià, A. (2018). Las trayectorias educativas de hombres y mujeres jóvenes. Una aproximación desde el análisis de secuencias. Papers, 103(1), 13-28. https://doi.org/10.5565/rev/papers.2290

Martínez García, J. S. (2007). Clase social, género y desigualdad de oportunidades educativas. Revista de Educación, 342, 287-306.

Martínez García, J. S. (2011). Género y origen social: Diferencias grandes en fracaso escolar administrativo y bajas en rendimiento educativo. Revista de la Asociación de Sociología de la Educación, 4(3), 270-285.

Ministerio de Educación y Formación Profesional (2020, 21 de enero). La tasa de abandono escolar en España alcanza su nivel más bajo desde que se tienen datos. Recuperado de https://bit.ly/2A2TjJD

Parrilla Latas, Á., Gallego Vega, C. y Moriña Díez, A. (2010). El complicado tránsito a la vida activa de jóvenes en riesgo de exclusión: una perspectiva biográfica. Revista de Educación, 351, 211 233.

Rodríguez Martínez, C. (2011). Género y cultura escolar. Madrid: Morata.

Rodríguez-Martínez, C. y Blanco García, N. (2015). Diferencias de género, abandono escolar y continuidad en los estudios. Revista Iberoamericana de Educación, 68, 59-78. Recuperado de https://rieoei.org/historico/documentos/rie68a03.pdf

Rodríguez San Julián, E. y Megías Quirós, I (2015). ¿Fuerte como papá? ¿sensible como mamá? Identidades de género en la adolescencia. Madrid: Centro Reina Sofía.

Rujas Martínez-Novillo, J. (2017). La construcción del «fracaso escolar» en España. Génesis y cristalización de un problema social. Papers, 102 (3), 477-507. http://dx.doi.org/10.5565/rev/papers.2297

Rumberger, . W. (2001). Why students drop out of school and what can be done. Conference on Dropouts in America: How severe is the problem? What do we know about intervention and prevention? Civil Rights Project. California: UCLA. Recuperado de https://escholarship.org/uc/item/58p2c3wp

Skelton, Ch. (2010). Gender and achievement: are girls the "success stories" of restructured education systems? Educational Review, 62(2), 131-142. doi: 10.1080/00131910903469536 